\title{
Poling and annealing of piezoelectric poly(vinylidene fluoride) micropillar arrays
}

\author{
I.O. Pariy $^{1}$, A.A. Ivanova ${ }^{1}$, V.V. Shvartsman ${ }^{2}$, M.A. Surmeneva ${ }^{1}$, R.A. Surmenev ${ }^{1 *}$ \\ ${ }^{1}$ Physical Materials Science and Composite Materials Centre, National Research Tomsk Polytechnic \\ University, 634050, Tomsk, Russia \\ *rsurmenev@mail.ru \\ ${ }^{2}$ Institute for Materials Science and Center for Nanointegration Duisburg-Essen (CENIDE), University of \\ Duisburg-Essen, 45141, Essen, Germany
}

The effect of annealing and poling processes on the crystalline phase and piezoelectric properties of poly(vinylidene fluoride) (PVDF) micropillar arrays was investigated. PVDF micropillars were prepared by the imprinting method, heated and treated with high-voltage poling. The effect of the treatment conditions on the crystallization behaviour and the piezoelectric properties of the patterned PVDF films were investigated by differential scanning calorimetry (DSC), Fourier transform infrared spectroscopy (FTIR) and piezoresponse force microscopy (PFM). Figure 1 shows the PFM images for the poled and annealed PVDF micropillar arrays and the piezoelectric characterization of the PVDF micropillar arrays.

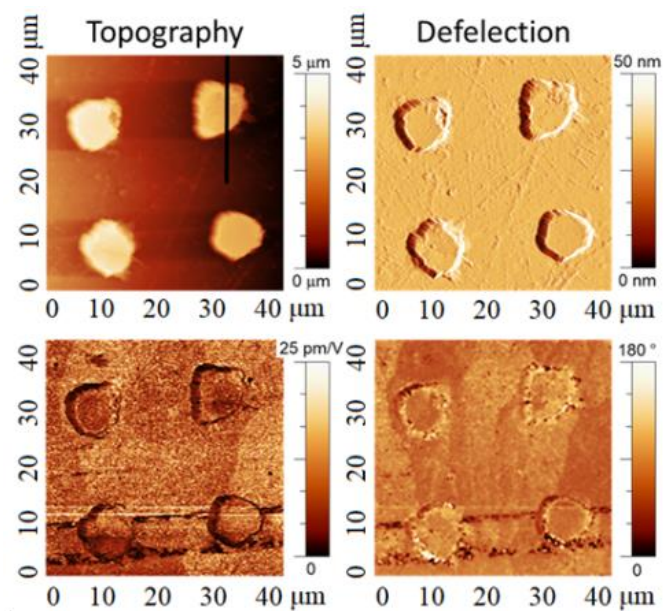

a

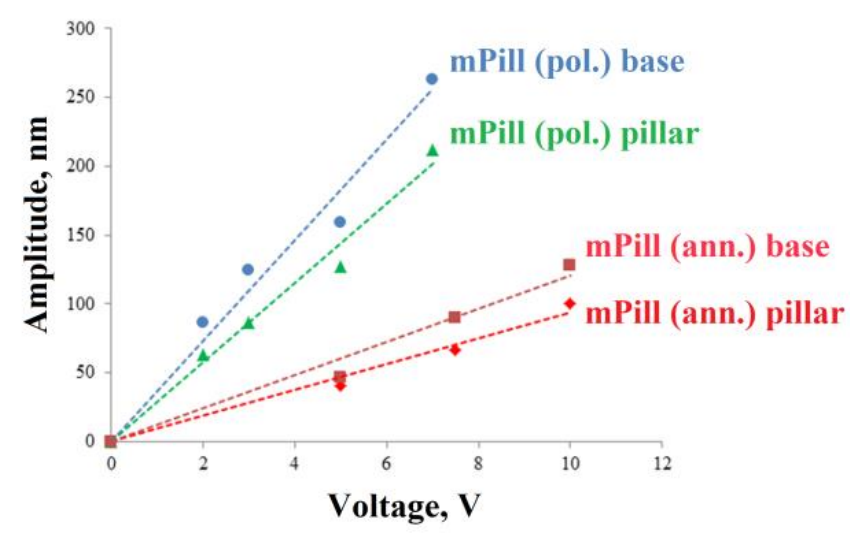

b

Figure 1. PFM images for the poled and annealed PVDF micropillar arrays on a $40 \times 40 \mu \mathrm{m}$ area (a) and piezoelectric characterization of the PVDF micropillar arrays (b).

The results of this study demonstrate a qualitative agreement of the $\beta$-phase content estimated using FTIR measurements, the degree of crystallinity obtained from DSC data and the intensity of the measured piezoelectric response, which was obtained from PFM analysis. Poled PVDF micropillars were shown to possess the highest crystallinity $(22.7 \%)$ and $\beta$-phase content (42\%) among all studied samples, which results in a 2.9-fold increase of the piezoelectric response. The FTIR measurements of poled and non-poled $\beta$-PVDF samples reveal that the main effect of the poling process is the partial rotation of molecular chains and the further transformation of the $\gamma$ into the $\beta$ phase, leading to an increase in the piezoelectric response, which was confirmed by the PFM results. The developed PVDF micropillar structures can be used as nanotip-based biosensors, nanotip-based tactile sensors, and power nanogenerators.

R.S. acknowledges the financial support from the Russian Science Foundation (project \#1873-10050). 